\title{
Quantum hydrodynamics in the rotating reference frame
}

\author{
Mariya Iv. Trukhanova a)
}

(Dated: 5 November 2018)

In this paper we apply quantum hydrodynamics (QHD) to study the quantum evolution of a system of spinning particles and particles that have the electric dipole moments EDM in the rotating reference frame. The method presented is based on the many-particle microscopic Schrodinger equation in the rotating reference frame. Fundamental QHD equations for charged or neutral spinning and EDM-bearing particles were shaped due to this method and contain the spin-dependent inertial force field. The polarization dynamics in systems of neutral particles in the rotating frame is shown to cause formation of a new type of waves, the dipole-inertial waves. We have analyzed elementary excitations in a system of neutral polarized fluids placed into an external electric field in $2 \mathrm{D}$ and $3 \mathrm{D}$ cases. We predict the novel type of $2 \mathrm{D}$ dipole-inertial wave and $3 \mathrm{D}$ - polarization wave modified by rotation in systems of particles with dipole-dipole interactions.

PACS numbers: 47.35.+i, 52.65.Kj, 77.22.Ej

Keywords: inertial forces, quantum hydrodynamic, dipole wave, inertial wave

\section{INTRODUCTION}

Over many years scientists paid attention to the effects occurring in the non-inertial frames. The influence of the inertial effects on electrons have always been the main focus of most studies, starting with those that belong to Barnett and Einstein - de Haas $\frac{1,2}{2}$. Recently the influence of rotation in spintronic applications has been noticed. The spin-dependent inertial force in an accelerating system under the presence of electromagnetic fields have been derived from the generally covariant Dirac equation ${ }^{3}$. It was shown that mechanical vibration in a high frequency resonator can create a spin current via the spin-orbit interaction augmented by the linear acceleration. The enhancement of the spin-rotation coupling due to the interband mixing was predicted in Ref. ${ }^{[4}$. The theoretical investigation of the inertial effects' influence on the spin dependent transport of conduction electrons in a semiconductor was reviewed in Ref.5. Equations of motion for a wavepacket of electrons in the two-dimensional planes subject to the spin-orbit interaction were derived in ${ }^{6}$, using the inertial effects due to the mechanical rotation. The author obtained a superposition of two cyclotron motions with different frequencies and a circular spin current.

A homogeneous fluid rotating with constant angular velocity leads to the emergence of an unusual class of inertial waves spreading in the interior of the fluid or inertial waves $^{7}[11$. Inertial waves, caused by fluid rotation, are circularly polarized waves with a sense of rotation fixed by their helicity. Inertial waves have many important features and play an important role in Geophysics 11,13 , in evolution of liquid planet core ${ }^{14]}, 15$, rotating stars ${ }^{16}$. The restoring force for such kind of waves in the rotating frame of reference is the Coriolis force, which leads

a) Faculty of physics, Lomonosov Moscow State University, Moscow, Russian Federation; Electronic mail: mar-tiv@yandex.ru to the circular motions of fluid elements. Large-scale geophysical and astrophysical flows are populated by a great variety of internal waves, some maintained by density stratification (internal gravity waves), some by the background planetary or stellar rotation (inertial waves), and yet others by the large-scale magnetic fields which thread through interplanetary space and are generated in the interiors of planets and stars (Alfven waves)

The investigation of inertial waves had been implemented in various geometries. The viscous flow inside a closed rotating cylinder of gas subjected to periodic axial compression had been investigated numerically 17 . An experimental study of inertial waves in a closed cone had been presented ${ }^{18}$, in which the inertial waves are excited by a slight periodic oscillation superimposed on the cone's basic rotation rate.

The experimental visualization of inertial waves had been realized using particle image velocimetry 19 . The author presented direct visualization of the velocity and vorticity fields in a plane normal to the rotation axis and determined the characteristic wavelength. The dynamics of the anisotropy of grid-generated decaying turbulence in a rotating frame had been experimentally investigated by means of particle image velocimetry on the large-scale "Coriolis" platform 20,21 .

The dipole-dipole interactions are the longest-range interactions possible between two neutral atoms or molecules and occur in many systems in nature. This interaction is one of the most important interactions between atoms or molecules. In recent years much attention is paid to the effect of the intrinsic electric dipole moment $(\mathrm{EDM})$ on the characteristics of charged and neutral particle systems. The propagation of perturbation of EDM does not require much energy as it occurs without mass transfer. This process may be used in the information transfer. In biological systems, for example, polarization processes, i.e. EDM propagation, are the predominant way of signal transfer ${ }^{22,23}$.

Dipole-dipole interactions, are studied and manipulated in Rydberg atoms, provide a strong coupling be- 
tween atoms in an ultracold Rydberg gas, because these atoms have high principal quantum number and have large electric transition dipole moments compared to ground state atoms 24,25 . This interaction can be tuned into resonance with a small electric field. This feature of Rydberg atoms can be used in quantum computing 26 , in the quantum information processing with cold neutral atoms27. Generation of entanglement between two individual Rydberg $R b^{87}$ atoms in hyperfine ground states had been observed in ${ }^{26}$ using Rydberg blockade effect. Dipole-dipole interactions between Rydberg atoms had been first observed by Raimond using spectral line broadening 28 . In the magneto-optical trap ground-state atoms are cooled down to $100 K$ by laser cooling 29 . Recently it has been shown that Rydberg excitation densities in a magneto-optical trap are limited by these interactions 30 . Another way is deceleration and trapping of Rydberg atoms by static electric fields31. Resonant electric dipole-dipole interactions between cold Rydberg atoms had been observed using the microwave spectroscopy ${ }^{32}$. The attraction of Rydberg atoms is that it is possible to tune the Rydberg energy levels through resonance for the dipoledipole energy transfer. The investigation of the properties of a cold $(100 \mathrm{~K})$ and dense $\left(\sim 10^{10} \mathrm{~cm}^{-3}\right)$ atomic Rydberg Cs gas had been studied in $\frac{\sqrt{33}}{3}$ using a "frozen Rydberg gas" model.

Classical methods were used previously to create a description of the collective dynamics of particles in the rotating frame that takes into account the inertial effects ${ }^{7}$ 10. We will use a quantum mechanics description for systems of $\mathrm{N}$ interacting particles is based upon the manyparticle Schrodinger equation (MPSE) that specifies a wave function in a $3 \mathrm{~N}$-dimensional configuration space of inertial frame. The many-particle quantum hydrodynamics in the inertial frame was investigated in Refs. 34 38. As wave processes, processes of information transfer, diffusion and other transport processes occur in the three-dimensional physical space of rotating frame, a need arises to turn to a mathematical method of physically observable values which are determined in a $3 \mathrm{D}$ physical space. To do so we should derive equations those determine dynamics of functions of three variables, starting from MPSE in the rotating frame. This problem has been solved with the creation of a method of manyparticle quantum hydrodynamics (MPQHD) in the noninertial frame.

\section{CONSTRUCTION OF FUNDAMENTAL EQUATIONS AND THE MODEL ACCEPTED}

In this section we derive the many-particle quantum hydrodynamics $(M P Q H G)$ equations from manyparticle Pauli-Schrodinger equation (MPSE). We receive the equations for the system of charged particles with spins. Method of MPQHG allows to present dynamic of system of interacting quantum particles in terms of functions defined in 3D physical space. It is important at investigation of wave process, which take place in 3D physical space.

For the beginning we present the one-particle Hamiltonian in the rotating frame which can be derived from the fundamental equation for spinning particle in a curve space-time ${ }^{3}$

$$
\left(\gamma^{\mu}\left[\partial_{\mu}-\Gamma_{\mu}-\frac{i q A_{\mu}}{\hbar}\right]+\frac{m c}{\hbar}\right) \psi=0,
$$

where $c, \hbar$ and $q$ are the speed of light, the Planck constant and charge of an electron respectively, $\Gamma_{\mu}$ is the spin connection ${ }^{3}$. The $4-$ spinor wave function $\psi$ contains the spin-up and spin-down components. The Hamiltonian for the system of charged particles contains the terms of the induced electric and magnetic fields due to inertial effect and can be obtained from the one-particle Dirac equation (1). The many-particle Hamiltonian in the rotating frame can be obtained as

$$
\begin{gathered}
\hat{H}=\hat{H}_{0}+\hat{H}_{\text {rotor }}+\hat{H}_{S O} \\
\hat{H}_{0}=\sum_{p=1}^{N}\left(\frac{\hat{D}_{p}^{2}}{2 m_{p}}+q_{p} \varphi_{p, e x t}-\mu_{p} \hat{\sigma}_{p}^{\alpha} B_{p, \text { ext }}^{\alpha}\right) \\
+\frac{1}{2} \sum_{p \neq n}^{N} q_{p} q_{n} G_{p n}-\frac{1}{2} \sum_{p \neq n, n}^{N} \mu_{p}^{2} F_{p n}^{\alpha \beta} \hat{\sigma}_{p}^{\alpha} \hat{\sigma}_{n}^{\beta}, \\
\hat{H}_{r o t o r}=-\sum_{p=1}^{N}\left(\varepsilon^{\alpha \beta \gamma} \Omega_{p}^{\alpha} \cdot r_{p}^{\beta} \hat{D}_{p}^{\gamma}+\frac{\hbar}{2} \hat{\sigma}_{p}^{\alpha} \cdot \Omega_{p}^{\alpha}\right), \\
\hat{H}_{S O}=-\sum_{p=1}^{N} \frac{\mu_{p}}{m_{p} c} \varepsilon^{\alpha \beta \gamma} \hat{\sigma}_{p}^{\alpha} \cdot E_{p, e x t}^{\beta} \hat{D}_{p}^{\gamma} \\
+\sum_{p \neq n}^{N} \frac{q_{p} \mu_{n}}{m_{n} c} \varepsilon^{\alpha \beta \gamma} \hat{\sigma}_{n}^{\alpha} \cdot \partial_{p}^{\beta} G_{p n} \hat{D}_{n}^{\gamma} \\
+\sum_{p=1}^{N} \frac{\mu_{p}}{m_{p} c} \varepsilon^{\alpha \beta \gamma} \varepsilon^{\beta \mu \nu} \varepsilon^{\nu i j} \hat{\sigma}_{p}^{\alpha} \cdot B_{p, e x t}^{\mu} r_{p}^{j} \Omega_{p}^{i} \hat{D}_{p}^{\gamma} .
\end{gathered}
$$

We consider a system of $N$ interacting particles. The following designations are used in the Hamiltonian (2): $\hat{\mathbf{D}}_{p}=-i \hbar \hat{\nabla}_{p}-\frac{q_{p}}{c} \mathbf{A}_{p}$, where $\mathbf{A}_{e x t}, \varphi_{p, e x t}$ - are the vector and scalar potentials of external electromagnetic field, $\mu_{p}=g \mu_{B} / 2, \mu_{p}$ - is the electron magnetic moment and $\mu_{p B}=q_{p} \hbar / 2 m_{p} c$ is the Bohr magneton, $q_{p}$ stands for the charge of electrons $q_{e}=-e$ or for the charge of ions 
$q_{p}=e$, and $\hbar$ is the Planck constant, $g \simeq 2.0023193, m_{p}$ denotes the mass of particles, $\mathrm{c}$ is the speed of light in vacuum. In this case the many-particle Pauli-Schrodinger equation for quantum particles motion in the external fields in the rotating frame has the form

$$
i \hbar \frac{\partial \Psi(R, t)}{\partial t}=\hat{H} \Psi(R, t)
$$

where $R=\left(\vec{r}_{1}, \ldots, \vec{r}_{N}\right)$ and $\Psi(R, t)$ is the rank $-N$ spinor. The first term at the right-hand side of the (3) gives sum of kinetic energies of all particles, it contains long derivative including action of the vortex part of the external electromagnetic field on the particle charge. The second term in 3 describes potential energy of charges in the external electric field. The third term describes the Zeeman effect with an external magnetic field and represents the Zeeman energy. The fourth term in (3) presents the Coulomb interaction between particles and the last term characterizes the spin-spin interactions. Green functions of the Coulomb and spin - spin interactions are $G_{p n}=1 / r_{p n}, \quad F_{p n}^{\alpha \beta}=4 \pi \delta_{\alpha \beta} \delta\left(\vec{r}_{p n}\right)+\partial_{p}^{\alpha} \partial_{n}^{\beta}\left(1 / r_{j k}\right)$. The Hamiltonian (4) characterizes the inertial effects. The first term in (4) describes the mechanical rotation coupling with the angular momentum of particles $\mathbf{r}_{p} \times \hat{\mathbf{D}}_{p}$ and leads to the Euler, Coriolis and centrifugal forces in the equation of motion. The second term is the spinrotation coupling and results in the Barnett effect. The spin-rotation coupling can be unified with the Zeeman energy $\mu_{p} \hat{\sigma}_{p} \cdot\left(\mathbf{B}_{p}+\mathbf{B}_{\Omega}\right)$ using the effective Barnett field of the particle $\mathbf{B}_{\Omega}=c m_{p} \boldsymbol{\Omega} / q_{p}$.

The spin-orbit coupling is characterized by the terms of Hamiltonian (5). The first term on the right-hand side represents the interaction of internal angular momentum or spin with the external electric field and the second term describes the effect of the Coulomb electric field on the spin. The third term in the Hamiltonian (5) describes the influence of the effective electric field on the moving spin in the inertial frame. The effective spin-dependent electric field arises in the rotating frame.

The first step in the construction of MQHD apparatus for the inertial frame is to determine the concentration of particles in the neighborhood of $\mathbf{r}$ in a physical space. If we define the concentration of particles as quantum average of the concentration operator in the coordinate representation $\hat{n}=\sum_{p}^{N} \delta\left(\mathbf{r}-\mathbf{r}_{p}\right)$ we arrive at the conclusive definition for the concentration

$$
n(\mathbf{r}, t)=\int d R \sum_{p}^{N} \delta\left(\mathbf{r}-\mathbf{r}_{p}\right) \Psi^{+}(R, t) \Psi(R, t)=\left\langle\Psi^{+} \Psi\right\rangle .
$$

We will use the definition for the average value of the physical quantity $f(R, t)$ of the particles

$$
\langle f\rangle=\int d R \sum_{p}^{N} \delta\left(\mathbf{r}-\mathbf{r}_{p}\right) f(R, t)
$$

Differentiation of $n(\mathbf{r}, t)$ with respect to time and applying of the Pauli-Schrodinger equation with Hamiltonian (2) leads to continuity equation in the rotating frame

$$
\partial_{t} n(\mathbf{r}, t)+\nabla \mathbf{j}(\mathbf{r}, t)=0
$$

where the current density vector takes a form of

$$
\mathbf{j}(\mathbf{r}, t)=\left\langle\frac{1}{2 m_{p}}\left(\left(\hat{\mathbf{j}}_{p} \Psi\right)^{+} \Psi+\Psi^{+} \hat{\mathbf{j}}_{p} \Psi\right)\right\rangle
$$

In the definition for the current density vector $(10)$ we use the current operator of i-th particle in the form

$$
\begin{gathered}
\hat{\mathbf{j}}_{p}=\hat{\mathbf{D}}_{p}-m_{p} \boldsymbol{\Omega} \times \mathbf{r}_{p}-\frac{\mu_{p}}{c} \hat{\sigma}_{p} \times \\
\times\left(\mathbf{E}_{p, e x t}+\frac{\left(\boldsymbol{\Omega} \times \mathbf{r}_{p}\right)}{c} \times \mathbf{B}_{p}\right)+\frac{1}{2} \sum_{p \neq n}^{N} \frac{q_{p}}{c} \mu_{p} \varepsilon^{\alpha \beta \gamma} \hat{\sigma}_{p}^{\beta} \nabla_{p}^{\gamma} G_{p n} .
\end{gathered}
$$

We derive the velocity of $\mathrm{i}$-th particle $\mathbf{v}_{p}$ is determined by equation

$$
\mathbf{v}_{p}=\frac{1}{m_{p}}\left(\nabla_{p} S-\frac{q_{p}}{c} \mathbf{A}_{p}\right)-\boldsymbol{\Omega} \times \mathbf{r}_{p}-\frac{\mu_{p}}{c m} \hat{\sigma}_{p} \times \mathbf{E}_{p, e f f}-\frac{i \hbar}{m_{p}} \phi^{+} \nabla_{p} \phi
$$

where the second term in the definition $(12)$ is the rotational velocity characterizing the effect of mechanical rotation. The effective electric field $\mathbf{E}_{\text {eff }}$ consists of the external electric field, the Coulomb internal field and the internal electric field originating from the mechanical rotation. It was shown that this field effects due to the spin-orbit interaction SOI with the mechanical rotation and can be displayed in the large SOI systems. In general case $\mathbf{v}_{p}(R, t)$ depends on the coordinate of all particles of the system $R$, where $R$ is the totality of $3 N$ coordinates of $N$ particles of the system $R=\left(\mathbf{r}_{1}, \ldots, \mathbf{r}_{N}\right)$. The $S(R, t)$ value in the formula 12 represents the phase of the wave function 


$$
\Psi(R, t)=a(R, t) \exp \left(\frac{i S(R, t)}{\hbar}\right) \phi(R, t)
$$

where $\phi$, normalized such that $\phi^{+} \phi=1$, is the new spinor. Velocity field $\mathbf{v}(\mathbf{r}, t)$ is the velocity of the local centre of mass in the rotating frame, is measured in the inertial frame and indicates a quantity measured in the rotating frame

$$
\mathbf{j}(\mathbf{r}, t)=n(\mathbf{r}, t) \mathbf{v}(\mathbf{r}, t)
$$

where the quantum equivalent of the thermal speed is determined as $\mathbf{u}_{p}(\mathbf{r}, R, t)=\mathbf{v}_{p}(R, t)-\mathbf{v}(\mathbf{r}, t)$. We differentiate the momentum density 10 with respect to time and apply the many-particle Pauli-Schrodinger equation to time derivatives of the wave functions $\Psi(R, t)$. As a result, a momentum balance equation can be obtained in the form

$$
\partial_{t} \mathbf{j}(\mathbf{r}, t)+\frac{1}{m} \partial_{\beta} \boldsymbol{\Pi}^{\beta}(\mathbf{r}, t)=\mathbf{F},
$$

where

$$
\boldsymbol{\Pi}^{\beta}(\mathbf{r}, t)=\left\langle\frac{1}{4 m_{p}}\left(\left(\hat{\mathbf{j}}_{p} \Psi\right)^{+} \hat{j}_{p}^{\beta} \Psi+\Psi^{+}\left(\hat{\mathbf{j}}_{p} \hat{j}_{p}^{\beta} \Psi\right)+c . c .\right)\right\rangle
$$

represents the momentum current density tensor and $\mathbf{F}$ represents a force field including the Coriolis, centrifugal and Euler forces in the rotating frame. We introduce the separation of particles thermal movement with velocities $\mathbf{u}_{p}(\mathbf{r}, R, t)$ and the collective movement of particles with velocity $\mathbf{v}(\mathbf{r}, t)$ in equations of continuity 9 and of the momentum balance (15). For that we substituted the wave function (13) in the definition of the basic hydrodynamical quantities. The momentum current density tensor 34 will has the new form of

$$
\begin{aligned}
\mathbf{\Pi}^{\beta}(\mathbf{r}, t) & =m n(\mathbf{r}, t) \mathbf{v}(\mathbf{r}, t) v^{\beta}(\mathbf{r}, t) \\
+ & \mathbf{p}_{\text {thermal }}^{\beta}(\mathbf{r}, t)+\mathbf{\Upsilon}_{\text {quantum }}^{\beta}(\mathbf{r}, t) .
\end{aligned}
$$

As we can see, the kinetic pressure tensor and quantum pressure tensor appear in the definition for the momentum current density tensor.

$$
\mathbf{p}_{\text {thermal }}^{\beta}(\mathbf{r}, t)=\left\langle a^{2} m_{p} \cdot \mathbf{u}_{p} u_{p}^{\beta}\right\rangle
$$

is the tensor of kinetic pressure and

$$
\mathbf{\Upsilon}_{\text {quantum }}^{\beta}(\mathbf{r}, t)=\left\langle-a^{2} \frac{\hbar^{2}}{2 m_{p}} \frac{\partial^{2} \ln a}{\partial \mathbf{r}_{p} \partial r_{p}^{\beta}}+\frac{\hbar^{2} a^{2}}{4 m_{p}} \nabla \mathbf{s}_{p} \cdot \nabla^{\beta} \mathbf{s}_{p}\right\rangle
$$

where the first term is the Madelung quantum potential. This tensor is proportional to $\hbar^{2}$, has a purely quantum origin and can therefore be interpreted as an additional quantum pressure. The second term characterizes the force produced by the self-interactions of the spins $\mathbf{s}_{p}$.

Taken in the approximation of self-consistent field, the continuity equation and momentum balance equation in the rotating frame have a form

$$
\begin{gathered}
\partial_{t} n(\mathbf{r}, t)+\nabla(n \mathbf{v})(\mathbf{r}, t)=0 \\
m n(\mathbf{r}, t)\left(\partial_{t}+\mathbf{v} \nabla\right) \mathbf{v}(\mathbf{r}, t)=e n(\mathbf{r}, t) \mathbf{E}(\mathbf{r}, t)+\frac{e}{c} n(\mathbf{r}, t) \mathbf{v}(\mathbf{r}, t) \times \mathbf{B}(\mathbf{r}, t)-\nabla_{\beta} \mathbf{p}^{\beta}(\mathbf{r}, t) \\
-\frac{\hbar^{2}}{2 m} n(\mathbf{r}, t) \nabla\left(\frac{\triangle \sqrt{n(\mathbf{r}, t)}}{\sqrt{n(\mathbf{r}, t)}}\right)+\mu n(\mathbf{r}, t) s_{\beta}(\mathbf{r}, t) \nabla B^{\beta}(\mathbf{r}, t)-\frac{\hbar^{2}}{4 m} \partial_{\beta}\left(n \nabla \mathbf{s}(\mathbf{r}, t) \cdot \partial^{\beta} \mathbf{s}(\mathbf{r}, t)\right) \\
+\frac{\hbar}{2} n(\mathbf{r}, t) s_{\beta}(\mathbf{r}, t) \nabla \Omega^{\beta}(\mathbf{r}, t)+\mathbf{F}_{\text {inertial }}(\mathbf{r}, t)+\mathbf{F}_{S O}(\mathbf{r}, t),
\end{gathered}
$$

where $n, m$ and $\mathbf{v}$ denote the density, mass and fluid velocity, $\mathbf{E}$ and $\mathbf{B}$ are the electric and magnetic fields in the rotating frame, $\mathbf{p}^{\beta}$ is the thermal pressure tensor, $\mathbf{s}^{2}=1-<a^{2} \xi_{p} \cdot \xi_{p}>/ n$ is the macroscopic spin angu- lar momentum, where $\xi_{p}$ is the thermal fluctuations of the spin about the macroscopic average. The first two terms describe the interaction with external electromagnetic field. As we can see, the transformations to the ro- 
tating frame of reference does not change the continuity equation (20). The first term at the right side of the equation (21) represents the effect of external electric field on the charge density and the second term represents the Lorentz force in the rotating frame. The fourth term at the right side of the equation (21) is a quantum force produced by quantum Madelung potential. The fifth term is the effect of non-uniform magnetic field on the magnetic moment. The fifth term appears in the equation of motion (21) through the magnetization energy and represents the Stern-Gerlach force due to the coupling between magnetic moment and magnetic field. The sixth term at the right side of the equation (21) is the spin force, produced by the spin stress 38 . The spin stress in the oneparticle model was derived by Takabayasi ${ }^{39}$. This development was performed using the postulate that a corpuscle of mass is embedded in the spinor wave. Spinor, in that way, is represented in terms of the spacetimedependent Euler angles which define the orientation of the triad relative to the fixed set of Cartesian axes 40,41 . In the context of this representation the spinor wave must constitute a new form of physical field that affects on the corpuscle of mass moving within it.

The seventh term on the right side of equation (21) represents the spin-rotation coupling. The eighth term is the inertial force density in the rotating frame

$\mathbf{F}_{\text {inertial }}(\mathbf{r}, t)=-2 m \boldsymbol{\Omega} \times n(\mathbf{r}, t) \mathbf{v}(\mathbf{r}, t)-m \frac{\partial \boldsymbol{\Omega}}{\partial t} \times \mathbf{P}(\mathbf{r}, t)$

$$
-m \boldsymbol{\Omega} \times(\boldsymbol{\Omega} \times \mathbf{P}(\mathbf{r}, t))-m \nabla_{\beta} \boldsymbol{\Omega} \times \boldsymbol{\Lambda}^{\beta}(\mathbf{r}, t),
$$

where the displacement vector

$$
\mathbf{P}(\mathbf{r}, t)=\left\langle\Psi^{+} \mathbf{r}_{p} \Psi\right\rangle
$$

and

$$
\boldsymbol{\Lambda}^{\beta}(\mathbf{r}, t)=\left\langle\frac{1}{2 m_{p}} \mathbf{r}_{p}\left(\left(\hat{j}_{p}^{\beta} \Psi\right)^{+} \Psi+\Psi^{+} \hat{j}_{p}^{\beta} \Psi\right)\right\rangle .
$$

The force field 22 depends on the mechanical rotation velocity $\boldsymbol{\Omega}$ and leads from the coupling between the mechanical rotation and angular momentum (4). The first term at the right side of the 22 is the Coriolis force density, the third term represents the centrifugal force density, the second and forth terms form the Euler force field density. The main features of the Coriolis force that this force cannot do work on the fluid and strives to deflect a fluid particle in a direction perpendicular to its instantaneous velocity. The last term on the right side of equation (21) is the spin-orbit force field density.

\section{A. Equation for the evolution of displacement vector}

To close the QHD equations set (20), (21) we derive equation for the displacement evolution. If we differentiate the definition for displacement (23) with respect to time and apply the Schrodinger equation, the required equation for the displacement evolution can be obtained

$$
\partial_{t} \mathbf{P}(\mathbf{r}, t)+\partial_{\beta} \mathbf{\Lambda}^{\beta}(\mathbf{r}, t)=0,
$$

We have two ways to close the QHD equations set. The first one is to express $\boldsymbol{\Lambda}^{\beta}$ in terms of $n, v$ and $\mathbf{P}$ using additional assumptions or experimental data. The other way is to derive the equation for evolution $\boldsymbol{\Lambda}^{\beta}$ in the same fashion it was accomplished previously for other material fields. Now the evolution equation $\boldsymbol{\Lambda}^{\beta}$ occurs in the form of

$$
\begin{array}{r}
\partial_{t} \boldsymbol{\Lambda}^{\beta}(\mathbf{r}, t)+\frac{1}{m} \partial_{\gamma} \boldsymbol{\Lambda}^{\beta \gamma}(\mathbf{r}, t)=\frac{e}{m} \mathbf{P}(\mathbf{r}, t) E_{e x t}^{\beta}(\mathbf{r}, t)+\frac{e}{m c} \epsilon^{\beta \gamma \delta} \boldsymbol{\Lambda}_{\beta}(\mathbf{r}, t) B^{\delta}(\mathbf{r}, t)-2 \boldsymbol{\Omega} \times \boldsymbol{\Lambda}(\mathbf{r}, t) \\
-\boldsymbol{\Omega} \times\left(\boldsymbol{\Omega} \times \mathbf{P}(\mathbf{r}, t) \cdot \frac{P^{\alpha}(\mathbf{r}, t)}{n(\mathbf{r}, t)}\right)-\partial_{t} \boldsymbol{\Omega} \times \mathbf{P}(\mathbf{r}, t) \frac{P^{\alpha}(\mathbf{r}, t)}{n(\mathbf{r}, t)}
\end{array}
$$

The first two terms describe the interaction of particles with external electromagnetic field. The next terms represent the inertial forces.

\section{B. Equation for the spin evolution}

To close the equations set 20 and 21 we derive equation for the spin evolution. If we differentiate the definition for spin-polarization 
$\mathbf{s}(\mathbf{r}, t)=\left\langle\phi^{+} \hat{\sigma}_{p} \phi\right\rangle$ with respect to time and apply the Pauli-Schrodinger equation with the Hamiltonian in the rotating frame, the required equation for the spin evolution $\mathbf{s}$ without thermal effects can be obtained

$$
\begin{gathered}
\left(\partial_{t}+\mathbf{v} \nabla\right) \mathbf{s}(\mathbf{r}, t)=\frac{2 \mu}{\hbar} \mathbf{s}(\mathbf{r}, t) \times \mathbf{B}(\mathbf{r}, t)+\mathbf{s}(\mathbf{r}, t) \times \mathbf{\Omega}(\mathbf{r}, t) \\
+\frac{\hbar}{2 m n(\mathbf{r}, t)} \mathbf{s}(\mathbf{r}, t) \times \partial_{\beta}\left(n(\mathbf{r}, t) \cdot \partial^{\beta} \mathbf{s}(\mathbf{r}, t)\right)-\frac{2 \mu}{\hbar c n(\mathbf{r}, t)} \varepsilon^{\alpha \beta \gamma} \varepsilon_{\beta \mu \nu} E_{e f f}^{\mu}(\mathbf{r}, t) J_{M}^{\gamma \nu}(\mathbf{r}, t)
\end{gathered}
$$

The first term at the right side of the equation (27) represents the torque caused by the interaction with the external magnetic field and the magnetic field of the spinspin interparticle interactions in the rotating frame. The second term is the spin rotation coupling term in the rotating frame and can be interpreted as the torque caused by the interaction with effective magnetic field $\mathbf{B}_{\Omega}$ introducing the Barnett effect to the right side of equation (27). The third term is the spin torque ${ }^{39}$. The fourth term characterizes the torque resulting from spin-orbit coupling in the effective electric field. The magnetic moment flux tensor occurs in this equation (27) in the form

$$
\mathbf{J}_{M}^{\beta}(\mathbf{r}, t)=\left\langle\frac{1}{4 m_{p}}\left(\Psi^{+} \hat{\sigma}_{p} \hat{j}_{p}^{\beta} \Psi+\left(\hat{\sigma}_{p} \hat{j}_{p}^{\beta} \Psi\right)^{+} \Psi\right)\right\rangle .
$$

We introduce the thermal fluctuation $\mathbf{w}_{p}(\mathbf{r}, R, t)=$ $\mathbf{s}_{p}(R, t)-\mathbf{s}(\mathbf{r}, t)$ of the spin about the macroscopic average $\mathbf{s}(\mathbf{r}, t)$ which determined in the neighborhood of $\mathbf{r}$ in a physical space. Using the Madelung decomposition of the N-particle wave function (13) we have obtained the definition for the magnetic moment flux tensor in the rotating frame

$$
\begin{array}{r}
\mathbf{J}_{M}^{\beta}(\mathbf{r}, t)=\mathbf{s}(\mathbf{r}, t) n(\mathbf{r}, t) v^{\beta}+\mathbf{j}_{\text {thermal }}^{\beta}(\mathbf{r}, t) \\
-\frac{\hbar}{2 m} n(\mathbf{r}, t) \mathbf{s}(\mathbf{r}, t) \times \partial_{\beta} \mathbf{s}(\mathbf{r}, t)
\end{array}
$$

where we introduce the definition for the thermal flux density tensor

$$
\mathbf{j}_{\text {thermal }}^{\beta}(\mathbf{r}, t)=\left\langle a^{2} \cdot \mathbf{s}_{p} u_{p}^{\beta}\right\rangle .
$$

We have two ways to close the equations set. The first one is to express $\mathbf{J}_{M}^{\beta}(\mathbf{r}, t)$ in terms of $n(\mathbf{r}, t), \mathbf{v}(\mathbf{r}, t)$ and $\mathbf{s}(\mathbf{r}, t)$ using additional assumptions or experimental data. The other way is to derive the equation for evolution $\mathbf{J}_{M}^{\beta}$ in the same fashion it was accomplished previously for other material fields.

\section{The evolution of polarization in the rotating frame}

The evolution of polarization for the particles with the electric dipole moment (EDM) can be derived using a method of quantum hydrodynamics. We receive the equations for the system of charged particles with EDM. We research the fluid of EDM-having polar particles (polar molecules). Obtaining equations could be used for neutral particles with the EDM as well. Method of quantum hydrodynamics allows to present dynamic of system of interacting quantum particles in terms of functions defined in 3D physical space in the rotating reference frame. The many-particle Hamiltonian for the EDM-having particles in the rotating reference frame has the form

$$
\begin{gathered}
\hat{H}=\hat{H}_{0}+\hat{H}_{\text {rotor }} \\
\hat{H}_{0}=\sum_{p=1}^{N}\left(\frac{\hat{\mathbf{D}}_{p}^{2}}{2 m_{p}}+q_{p} \varphi_{p, e x t}-\mathbf{d}_{p} \mathbf{E}_{p, e x t}\right) \\
+\frac{1}{2} \sum_{p \neq n}^{N} q_{p} q_{n} G_{p n}-\frac{1}{2} \sum_{p \neq n, n}^{N} G_{p n}^{\alpha \beta} \hat{d}_{p}^{\alpha} \hat{d}_{n}^{\beta}, \\
\hat{H}_{\text {rotor }}=-\sum_{p=1}^{N}\left(\varepsilon^{\alpha \beta \gamma} \Omega_{p}^{\alpha} \cdot r_{p}^{\beta} \hat{D}_{p}^{\gamma}+\frac{\hbar}{2} \hat{\sigma}_{p}^{\alpha} \cdot \Omega_{p}^{\alpha}\right) .
\end{gathered}
$$

The new third term in $(32)$ is considered in the Hamiltonian of particles through the dipole energy in the external electric field. The fourth term in (32) presents the Coulomb interaction between charged particles and the last term characterizes the dipole-dipole interactions between dipoles, where $G_{p n}^{\alpha \beta}=\partial_{p}^{\alpha} \partial_{n}^{\beta} / r_{p n}$.

From the many-particle Hamiltonian (31) the required equation for the polarization evolution can be obtained as 36 


$$
\partial_{t} \mathbf{D}(\mathbf{r}, t)+\partial_{\beta} \mathbf{R}^{\beta}(\mathbf{r}, t)=0,
$$

where the polarization vector field of the EDM-having particles has the form

$$
\mathbf{D}(\mathbf{r}, t)=\left\langle\Psi^{+} \mathbf{d}_{p} \Psi\right\rangle,
$$

and a polarization current can be derived in the form

$$
\mathbf{R}^{\beta}(\mathbf{r}, t)=\left\langle\frac{\mathbf{d}_{p}}{2 m_{p}}\left(\hat{j}_{p}^{+\beta} \Psi^{+} \Psi+\Psi^{+} \hat{j}_{p}^{\beta} \Psi\right)\right\rangle .
$$

\section{WAVE OF POLARIZATION}

\section{A. The inertial frame}

We investigate the system of neutral particles resides in a uniform electromagnetic field. It is also assumed that interactions make the largest contribution into the changes in $\mathbf{R}^{\beta 36}$. If so then we use the equation (34) and the equation for the polarization current density evolution 36

$$
\begin{aligned}
\partial_{t} \mathbf{R}^{\beta}(\mathbf{r}, t) & =\sigma \frac{\mathbf{D}(\mathbf{r}, t) D^{\gamma}(\mathbf{r}, t)}{m n(\mathbf{r}, t)} \times \\
& \times \nabla_{\beta} \int d \mathbf{r}^{\prime} G^{\gamma \mu}\left(\mathbf{r}, \mathbf{r}^{\prime}\right) D_{\mu}\left(\mathbf{r}^{\prime}, t\right)
\end{aligned}
$$

The term on the right hand side of (37) characterizes the dipole-dipole interactions between the particles. This allows the analysis of polarization waves in a system of neutral particles. If we derive a solution for eigenwaves in a $2 \mathrm{D}$ system the dispersion equation has a form of

$$
\omega=\sqrt{\sigma \frac{\beta(k)}{m n_{0}}}|\kappa| E_{0} k^{3 / 2},
$$

where $\beta(k)$ is defined by the relation

$$
\beta(k)=2 \pi \int_{\xi}^{\infty} d r \frac{J_{0}(r)}{r^{2}},
$$

here $\xi=r_{0} k, r_{0}$ there is an ionic or molecular radius and $k=\sqrt{k_{x}^{2}+k_{y}^{2}}$ is a modulus of the wave vector. As $\lambda_{\min }=2 \pi / k_{\max }>2 r_{0}$ then $\xi \subset(0, \pi)$. The dispersion dependence (38) is presented on fig. 1(a)].

In $1 \mathrm{D}$ case $\omega(k)$ occurs as

$$
\omega=\sqrt{\frac{\sigma \beta_{1}(k)}{m n_{0}}}|\kappa| E_{0} k^{2},
$$

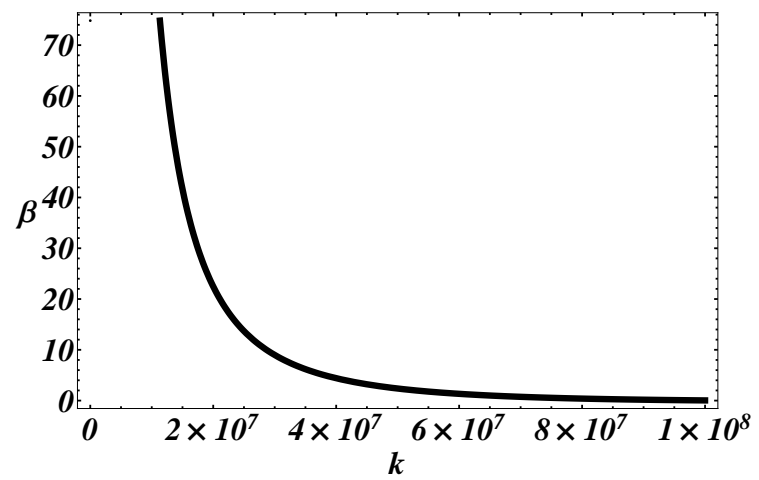

(a)

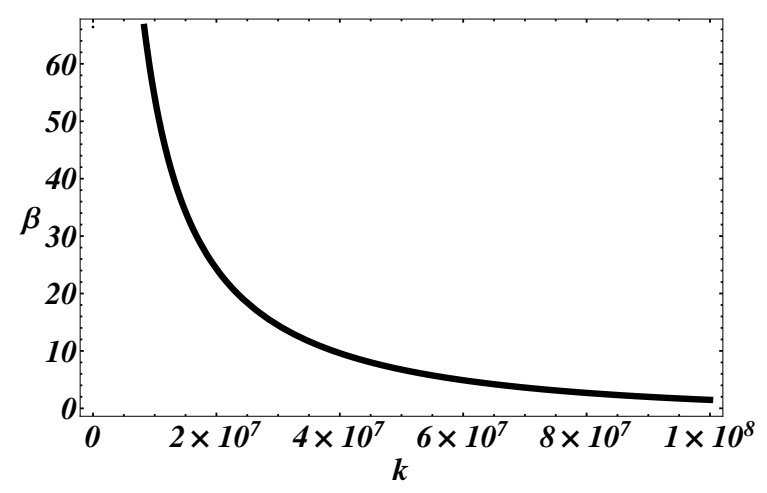

(b)

FIG. 1. Color online: The figure (a) presents of the variable $\beta_{1}(k)$ on the wave vector $\mathrm{k}$. Ionic or molecular radius $r_{0}$ are assumed to equal $0.1 \mathrm{~nm}$. The figure (b) presents the dependence of the variable $\beta(k)$ on the wave vector $\mathrm{k}$. Ionic or molecular radius $r_{0}$ is assumed to equal $0.1 \mathrm{~nm}$.

where

$$
\beta_{1}(k)=2 \int_{\xi}^{\infty} d r \frac{\cos (r)}{r^{3}} .
$$

The quantity (41) is presented on Figs. 1(a) $)$. The dispersion relations (38) and (40) characterize the waves of polarization in the system of neutral particles with dipole moments. This waves exist on a level with the acoustic waves. Equations of continuity (20) and of the momentum balance (21) herein describe the dynamics of the acoustic wave. Dispersion branches of a novel type that occurs due to the polarization dynamics were discovered in various physical systems 36 . The waves of electric polarization we discovered possess the following feature their frequencies $\omega$ tends to zero provided that $k \rightarrow 0$.

\section{B. Dipole-inertial wave}

\section{2D-eigenwaves}

In this section we consider the fluid of neutral particles in the rotating reference frame. We derive disper- 


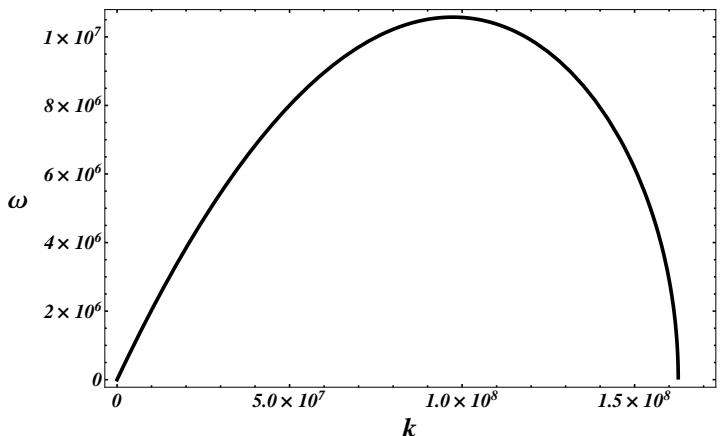

(a)

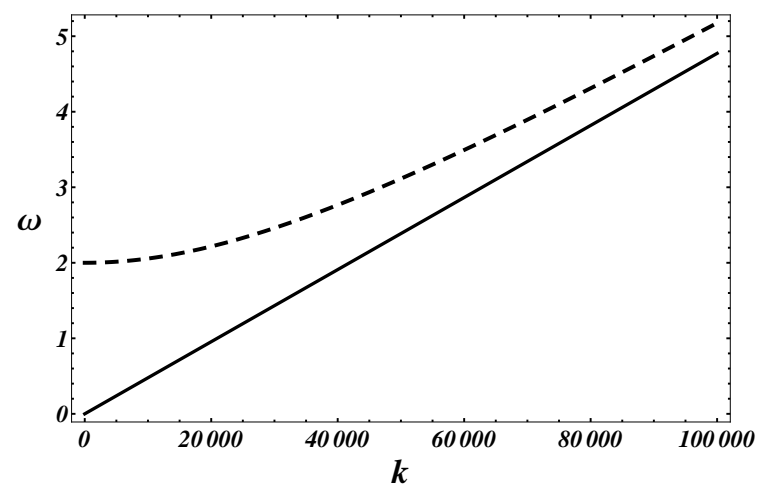

(b)

FIG. 2. Color online: The dependence of frequency $\omega$ on the wave vector $k$ is displayed for the case of two-dimension polarization mode which dispersion characteristic is defined by the equation (38) for the figure (a) and of two-dimension polarization mode modified by rotation for the figure (b). The dashed branch describes the polarization wave modified by rotation or dipole-inertial $2 \mathrm{D}$-mode (43). The radius $r_{0}$ is supposed to be $0.1 \mathrm{~nm}$. Equilibrium polarization has form $D_{0}=\kappa E_{0}$. Static electric permeability $\kappa$ is defined by the equation $\kappa=n_{0} d_{0}^{2} /\left(3 k_{B} T\right)$, where $d_{0}$ - is a dipole moment of an molecule, $T$ - temperature of the medium, $k_{B}$ - Boltzmann constant. System parameters are assumed to be as follows: $n_{0}=10^{12} \mathrm{sm}^{-} 2, d_{0} \simeq 0.16 D, T=100 K, E_{0} \simeq 10^{2} \mathrm{~V} / \mathrm{m}$ and $m=10^{-23} \mathrm{~g}$.

sion characteristics of eigenwaves in 2D systems of neutral particles that take account of the EDM collective dynamics. The particles are assumed here to localize on a $x y$ - plane and reside in a uniform electromagnetic field orthogonal to the plane $x y$. For simplicity the centrifugal force is neglected and only the effects of the Coriolis force are taken into consideration $|\boldsymbol{\Omega} \times(\boldsymbol{\Omega} \times \mathbf{P})|<<2|n \boldsymbol{\Omega} \times \mathbf{v}|$ the equation 37 transforms into

$$
\begin{gathered}
\partial_{t} \mathbf{R}^{\alpha}(\mathbf{r}, t)=\sigma \frac{D^{\alpha}(\mathbf{r}, t) D^{\gamma}(\mathbf{r}, t)}{m n(\mathbf{r}, t)} \times \\
\times \nabla \int d \mathbf{r}^{\prime} G^{\mu \gamma}\left(\mathbf{r}, \mathbf{r}^{\prime}\right) D_{\mu}\left(\mathbf{r}^{\prime}, t\right)-2 \boldsymbol{\Omega} \times \mathbf{R}^{\alpha},
\end{gathered}
$$

and to close the equation 42 we use the equation for the polarization evolution (34). If we derive a solution for eigenwaves in a $2 \mathrm{D}$ system the dispersion equation has a form of

$$
\omega=\sqrt{\sigma \frac{\beta(k)}{m n_{0}}|\kappa|^{2} E_{0}^{2} k^{3}+4 \Omega^{2}},
$$

the dispersion relation characterizes the 2D-polarization wave modified by rotation (fig. 2(b) , where the $z$ axis is directed along the rotation axis $\boldsymbol{\Omega}=\Omega \mathbf{z}$.

\section{3D-wave}

To analyze 3D systems we use the set of equations (34) and the polarization current density equation. The equation for the polarization current density in the rotating frame and in the approximation of the self-consistent field has the form of

$$
\partial_{t} \mathbf{R}^{\alpha}=\sigma \frac{D^{\alpha} D^{\gamma}}{m n} \times \nabla E^{\gamma}-2 \boldsymbol{\Omega} \times \mathbf{R}^{\alpha},
$$

where the total electric field $\mathbf{E}$ leads from a field equation $\nabla \cdot \mathbf{E}=-4 \pi \nabla \cdot \mathbf{D}$. Here $z$ axis is directed along the rotation axis, $\mathbf{D}_{0}=D_{\|} \mathbf{z}$ and two-dimensional position vector $\mathbf{r}_{\perp}$ is directed in the $x y$ plane normal to the rotation axis, a wave number $k_{\|}$is the component of the three-dimension wave vector along the rotation axis and $\mathbf{k}_{\perp}$ is the wave vector in the $x y$ plane. These equations give the dispersion low

$$
\begin{gathered}
\omega^{2}=\frac{\Omega_{d}^{2}+4 \Omega^{2}}{2} \pm \\
\pm \sqrt{\left(\frac{\Omega_{d}^{2}}{2}+2 \Omega^{2}\right)^{2}-4 \Omega_{d}^{2} \Omega^{2} \cos ^{2} \theta}
\end{gathered}
$$

For a fluid at rest $(\Omega=0)$ this relation gives the frequencies the zero-frequency transverse mode of a fluid at rest $\omega=0$ and $\omega=\Omega_{d}$ corresponding to the transverse polarization wave. It seems interesting to analyze the effect of rotation on the wave propagation in different limits. Obviously, in the approximation of large wave vectors $k_{\|} \rightarrow \infty$ and $\Omega_{d}^{2}>>\Omega^{2}$ one branch of dispersion yields the inertial wave

$$
\omega^{2}=4 \Omega^{2} \frac{k_{\|}^{2}}{k^{2}},
$$

and another represents the polarization wave modified by rotation

$$
\omega^{2}=\sigma \frac{4 \pi D_{0}^{2}}{m n} k_{\|}^{2}-4 \Omega^{2} \frac{k_{\|}^{2}}{k^{2}} \rightarrow \Omega_{d}^{2}
$$




\section{Conclusions}

In this paper the method of QHD was developed for spin-bearing and EDM-bearing particles in the rotating frame. QHD equations are a consequence of MPSE in which particles interaction is directly taken into account. The system of QHD equations we constructed is comprised by equations of continuity (20), of the momentum balance (21) and of the spin evolution equation (27). Using the developed method we derived the inertial force field (22) which consists of the Coriolis force density, the centrifugal force density and of the Euler force field density. We close our system of equations by the displacement evolution equation (II A). In our studies of wave processes we used a self-consistent field approximation of the QHD equations. In this paper we also analyzed wave excitations caused by EDM dynamics in systems of charged and neutral particles in the rotating frame. For this purpose we derived the polarization evolution equation (34) and the polarization current for 2D systems (42) and 3D fluids (44). Waves in a 2D fluid of EDMbearing neutral particles in various physical dimensions were considered. The effect of mechanical rotation on the polarization dynamics was derived. Dispersion branches of a novel type that occurs due to polarization dynamics in the rotating frame were discovered for 2D (43) and 3D (45) physical systems with dipole-dipole interactions. Transfer of polarization disturbances plays a major role in the information transfer in biological systems. Such processes do not require particles of the medium to possess EDM as the dynamics of a system of charged particles leads to collective polarization.

${ }^{1}$ S. J. Barnett, Phys. Rev. 6, 239 (1915).

${ }^{2}$ A. Einstein and W. J. de Haas, Verh. Dtsch. Phys. Ges. 17, 152 (1915).

${ }^{3}$ Mamoru Matsuo, Junichi Ieda, Eiji Saitoh, and Sadamichi Maekawa, Spin-dependent inertial force and spin current in accelerating systems, Phys. Rev. B. 84104410 (2011)

${ }^{4}$ Mamoru Matsuo, Jun'ichi Ieda, and Sadamichi Maekawa Phys. Rev. B 87, 115301 (2013)

${ }^{5}$ B. Basu, Debashree Chowdhury, Inertial effect on spin orbit coupling and spin transport, Annals of Phys, 335, 47 (2013); Spin Transport in non-inertial frame, Physica B, 448, 155 (2014).

${ }^{6}$ Mamoru Matsuo, Junichi Ieda, Eiji Saitoh, Sadamichi Maekawa, Phys. Rev. Lett. 106, 076601 (2011)

${ }^{7}$ Edouard B. Sonin, Dynamics of Quantised Vortices in Superfluids, Cambridge University Press, London, (2016)

${ }^{8}$ P. A. Davidson, Turbulence in Rotating, Stratified and Electrically Conducting Fluids, Cambridge University Press, London, (2013)

${ }^{9}$ Greenspan H., The theory of rotating fluids, Cambridge University Press, London, (1968)

${ }^{10}$ Lighthill J., Waves in fluids, Cambridge University Press, London, (1978)

${ }^{11}$ Cushman-Roisin B., Introduction to geophysical fluid dynamics, Prentice-Hall, Englewood Cliffs, (1994)

${ }^{12}$ J. Boisson, D. Cebron, F. Moisy and P. P. Cortet, Earth rotation prevents exact solid body rotation of fluids in the laboratory, EPL, 98, 59002 (2012)

${ }^{13}$ Pedlosky J., Geophysical fluid dynamics, Springer, Heidelberg (1987)

${ }^{14}$ K. D. Aldridge and L. I. Lumb., Inertial waves identified in the earths fluid outer core. Nature, 325(6103):421 (1987)
${ }^{15} \mathrm{H}$. Bondi and R. A. Lyttleton, On the dynamical theory of the rotation of the earth. The effect of precession on the motion of the liquid core, Proceedings of the Cambridge Philosophical Society, 49(3):498, (1953)

${ }^{16}$ Wen Fu and Dong Lai, Corotational instability, magnetic resonances and global inertial-acoustic oscillations in magnetized black hole accretion discs, Mon. Not. R. Astron. Soc. 410, 399 (2011)

${ }^{17}$ Duguet Y., Oscillatory jets and instabilities in a rotating cylinder, Phys Fluids 18, 104104 (2006)

${ }^{18}$ Beardsley RC., An experimental study of inertial waves in a closed cone, Stud Appl Math XLIX(2):187196 (1970)

${ }^{19}$ L. Messio, C. Morize, M. Rabaud and F. Moisy, Experimental observation using particle image velocimetry of inertial waves in a rotating fluid, Exp. in Fluids 44, 519 (2008)

${ }^{20}$ C. Morize, F. Moisy, M. Rabaud and J. Sommeria, Dynamics of the anisotropy in decaying rotating turbulence with confinement effects, 18 - eme Congres Francais de Mecanique, Grenoble (2007).

${ }^{21}$ F. Moisy, C. Morize, M. Rabaud, J. Sommeria, Decay laws, anisotropy and cyclone-anticyclone asymmetry in decaying rotating turbulence J. Fluid Mech. 666, 5 (2011)

${ }^{22}$ Gregory S. Engel, Tessa R. Calhoun, Elizabeth L. Read, Tae-Kyu Ahn, Tomas Mancal, Yuan-Chung Cheng, Robert E. Blankenship, and Graham R. Fleming, Evidence for wavelike energy transfer through quantum coherence in photosynthetic systems, Nature 446 (2007), 782.

${ }^{23}$ Hohjai Lee, Yuan-Chung Cheng, and Graham R. Fleming, Coherence Dynamics in Photosynthesis: Protein Protection of Excitonic Coherence, Science 316 (2007), 1462.

${ }^{24}$ Kourosh Afrousheh, Observation of resonant electric DipoleDipole interactions between cold Rydberg atoms using microwave spectroscopy, Waterloo, Ontario, Canada (2006)

${ }^{25}$ H. J. Metcalf, and P. van der Straten, Laser cooling and trapping, Springer (1999).

${ }^{26}$ M. D. Lukin, M. Fleischhauer, R. Cote, L. M. Duan, D. Jaksch, J. I. Cirac, and P. Zoller, Phys. Rev. Lett. 87, 037901 (2001)

${ }^{27}$ M. S. Safronova, C. J. Williams, and C.W. Clark, Phys. Rev. A 67, 040303 (2003).

${ }^{28}$ J. M. Raimond, G. Vitrant, and S. Haroche, J. Phys. B 14, L655 (1981).

${ }^{29}$ I. Mourachko, D. Comparat, F. de Tomasi, A. Fioretti, P. Nosbaum, V. M. Akulin, and P. Pillet, Many-Body Effects in a Frozen Rydberg Gas, Phys. Rev. Lett. 80 (1998), 253.

${ }^{30}$ K. Singer, M. Reetz-Lamour, T. Amthor, L. G. Marcassa, and M. Weidemuller, Phys. Rev. Lett. 93, 163001 (2004).

${ }^{31}$ E. Vliegen, H. J. Worner, T. P. Softley, and F. Merkt, Nonhydrogenic Effects in the Deceleration of Rydberg Atoms in Inhomogeneous Electric Fields, Phys. Rev. Lett. 92 (2004), 033005.

${ }^{32}$ K. Afrousheh, P. Bohlouli-Zanjani, D. Vagale, A. Mugford, M. Fedorov, and J. D. D. Martin, PRL 93, 233001 (2004)

${ }^{33}$ I. Mourachko, D. Comparat, F. de Tomasi, A. Fioretti, P. Nosbaum, V. M. Akulin, and P. Pillet Phys. Rev. Lett. 80, 253 (1998)

${ }^{34}$ L. S. Kuzmenkov and S. G. Maksimov, Theoretical and Mathematical Physics, 118, 227 (1999)

${ }^{35}$ L. S. Kuzmenkov, S. G. Maksimov, and V. V. Fedoseev, Theoretical and Mathematical Physics, 126, 110 (2001)

${ }^{36}$ P. A. Andreev, L. S. Kuzmenkov, M. I. Trukhanova, Phys.Rev.B., A quantum hydrodynamics approach to the formation of new types of waves in polarized two-dimension systems of charged and neutral particles, 84, 245401 (2011)

${ }^{37}$ P. A. Andreev, M. I. Trukhanova, Russian Physics Journal 53, 1196 (2011)

${ }^{38}$ Mariya Iv. Trukhanova, Prog. Theor. Exp. Phys., Quantum Hydrodynamics Approach to The Research of Quantum Effects and Vorticity Evolution in Spin Quantum Plasmas, 2013, 111I01 (2013)

${ }^{39}$ T. Takabayasi, Prog. Theor. Phys. 14, 283 (1955);

T. Takabayasi, J. P. Vigier, Prog. Theor. Phys. 18, 573 (1957); 
T. Takabayasi, Prog. Theor. Phys. 70, 1 (1983);

T. Takabayasi, Prog. Theor. Phys. Suppl. 4, 1 (1957);

${ }^{40}$ P. R. Holland, P. N. Kyprianidis, Ann. Inst. Henri Poincare. 49, 325 (1988);

P. R. Holland, J. P. Vigier, Found. Phys. 18, 741 (1988);

Peter R. Holland, The Quantum Theory of Motion, Cambridge University Press, (1993);
P. R. Holland, Phys. Lett. A. 128, 9 (1988); P. R. Holland, Phys. Rep. 169, 293 (1988)

${ }^{41}$ H. A. Kramers, Quantentheorie des Electrons and des Strablung (Leipzig, 1938) 259 